\title{
Bulimia em adolescentes praticantes de atividade física
}

\author{
Bulimia in adolescents who practice physical activity \\ Bulimia en adolescentes que practican actividad fisica
}

Recebido: 07/05/2021 | Revisado: 28/05/2021 | Aceito: 29/05/2021 | Publicado: 01/06/2021

Natália Fernandes Henrique Santos

ORCID: https://orcid.org/0000-0001-7755-6064

Universidade Castelo Branco, Brasil

E-mail: nataliahenriquefs@gmail.com

\begin{abstract}
Resumo
A pressão social para ser magro tem feito com que milhões de adolescentes, na maioria do sexo feminino, utilizem métodos inadequados, como vômitos auto induzidos e a prática excessiva de exercícios físicos para conseguir alcançar o padrão de beleza idealizado pela mídia. Essa difusão deste padrão tem sido apontada como uma das causas de transtornos alimentares em adolescentes. Este trabalho revisa a literatura sobre bulimia nervosa não purgativa, discutindo suas causas, efeitos e terapia nutricional.
\end{abstract}

Palavras-chave: Transtorno Alimentar; Bulimia; Adolescente; Feminino; Atividade Física.

\begin{abstract}
The social pressure to be thin has caused millions of adolescents, mostly females, to use inappropriate methods, such as self-induced vomiting and excessive physical exercise to achieve the standard of beauty idealized by the media. This spread of this pattern has been identified as one of the causes of eating disorders in adolescents. This paper reviews the literature on non-purgative bulimia nervosa, discussing its causes, effects and nutritional therapy.
\end{abstract}

Keywords: Eating Disorder; Bulimia; Adolescent; Feminine; Physical Activity.

\section{Resumen}

La presión social por ser delgada ha provocado que millones de adolescentes, en su mayoría mujeres, utilicen métodos inapropiados, como los vómitos autoinducidos y el ejercicio físico excesivo, para alcanzar el estándar de belleza idealizado por los medios de comunicación. Esta propagación de este patrón se ha identificado como una de las causas de los trastornos alimentarios en los adolescentes. Este artículo revisa la literatura sobre la bulimia nerviosa no purgante, discutiendo sus causas, efectos y terapia nutricional.

Palabras clave: Desorden Alimenticio; Bulimia; Adolescente; Femenino; Actividad Física.

\section{Introdução}

$\mathrm{Na}$ atualidade, milhões de pessoas apresentam comportamentos disfuncionais caracterizados como transtornos alimentares. A grande maioria dos acometidos por estes distúrbios são adolescentes jovens do sexo feminino (Schimitz et al., 2009).

O modelo de beleza imposto pela sociedade atual corresponde a um corpo magro sem, contudo, considerar aspectos relacionados com a saúde e as diferentes constituições físicas da população (Oliveira et al.,2003). Entender o papel do corpo na atualidade é tarefa complicada, objeto de estudo de diversas áreas, levando a discussões profundas e complexas. O que é beleza? Quais atributos ditam o belo? Qual o papel da cultura, política, economia nesta definição? Em qual momento se nomeou o magro de belo? Segundo Baudrillard (1991), quando o corpo foi recuperado como instrumento de prestígio, tornou-se objeto de um trabalho de investimento, representando um esforço ainda mais alienado que a exploração do corpo na força de trabalho. Apareceu o pensamento de que ao não se cumprirem as devoções corporais, ao pecar por omissão, a pessoa seria castigada. A beleza se tornou, para a mulher, um imperativo absoluto e deixou de ser efeito da natureza e suplemento das qualidades morais. O autor afirma que a beleza constitui um imperativo tão absoluto, pelo simples fato de ser uma forma do capital: a ética da beleza reduz todos os valores concretos do corpo (energético, gestual e sexual) a um valor de permuta funcional. 
Research, Society and Development, v. 10, n. 6, e29110615874, 2021

(CC BY 4.0) | ISSN 2525-3409 | DOI: http://dx.doi.org/10.33448/rsd-v10i6.15874

A adolescência, que de acordo com a OMS compreende a faixa etária entre 10 e 19 anos, é um período de transformações biológicas e psicossociais, em que alterações do corpo ocorrem paralelamente à constituição da personalidade, refletindo assim no desenvolvimento afetivo-emocional (Dunker et al., 2009).

De acordo com Souto (2002), o início da adolescência é um período de busca de identidade, de forma que a preocupação é centrada no corpo e na aparência física. Por isso, ocorre a necessidade de readaptar-se à imagem corporal (modo de vivenciar o peso, o tamanho ou a forma corporal), uma vez que a construção dessa imagem pode alterar-se, tornando-se patológica e representando um risco para o desenvolvimento de um transtorno alimentar. Cordás e colaboradores (2004) afirmam que "[...] os transtornos alimentares são doenças psiquiátricas que afetam, na sua maioria, adolescentes e adultos jovens do sexo feminino, podendo levar a grandes prejuízos biológicos e psicológicos e aumento de morbidade e mortalidade". Para CLAUDINO \& BORGES (2002), entretanto, “[...] os transtornos alimentares são síndromes comportamentais cujos critérios têm sido amplamente estudados nos últimos 30 anos. São descritos como transtornos e não como doenças por ainda não se conhecer bem sua etiopatogenia".

Um dos transtornos alimentares que pode ser desenvolvido é a bulimia. Este transtorno alimentar pode ser dividido em purgativo, com o uso de laxantes, diuréticos e afins e não purgativo, fazendo uso excessivo de exercícios físicos (Carvalho et al., 2009). Para tal transtorno estima-se uma prevalência entre 3 e 5\% na população feminina adolescente e jovem adulta (Vale Amo, 2002; Castro \& Goldstein, 1995). Indivíduos com bulimia nervosa consomem grandes quantidades de alimento, especialmente se submetidos à situação de estresse. Para evitar o ganho de peso e aliviar a culpa e a vergonha provocadas pelo descontrole alimentar, apresentam comportamentos compensatórios. Devido ao caráter "secreto" desse ritual, a bulimia nervosa é de difícil diagnóstico, sobretudo em sua fase inicial, o que dificulta a prevenção e tratamento precoces (Castilla \& Bastin, 1998).

A atividade física pode ser um dos métodos utilizados pelos indivíduos com transtorno alimentar para perda/controle de peso, ocupando lugar dominante como estratégia para perda de peso (Beumont et al.,1984).

O presente trabalho tem por objetivo revisar a literatura sobre bulimia nervosa em adolescentes do sexo feminino que praticam atividade física, discutindo a influência da mídia nesses indivíduos, definições, efeitos e terapia nutricional.

\section{Metodologia}

O estudo consistiu em uma pesquisa bibliográfica de natureza qualitativa, cuja bibliografia sobre o tema publicado na última década foi obtida em bibliotecas situadas na Cidade do Rio de Janeiro e em bases de dados on-line, contendo livros e periódicos científicos. Com base nos autores Bittar (2020), Estrela (2018), Santos (2019), Nunes (1994), Córdas (2004) entre outros.

\section{Resultados e Discussão}

Há alguns anos, o modelo de beleza exaltado era caracterizado por um corpo feminino obeso. Porém, nos últimos anos, a figura humana ideal passou a ser um corpo magro, esguio e atlético (Nunes et al., 1994). Entretanto, esse padrão de beleza desconsidera os aspectos de saúde e as diferentes constituições físicas da população (Oliveira et al., 1993), acarretando, muitas vezes, uma imagem corporal negativa, indicada por altos níveis de insatisfação com o corpo (Jones et al., 2004).

O termo Imagem Corporal vem sendo usados frequentemente tendo como foco de investigação o corpo humano. Tavares (2003) diz que a compreensão do conceito de Imagem Corporal está vinculada ao significado dos termos imagens e corpo e que sua definição não é simplesmente uma questão de linguagem, tem uma dimensão muito maior, se pensarmos na subjetividade de cada indivíduo. 
Research, Society and Development, v. 10, n. 6, e29110615874, 2021

(CC BY 4.0) | ISSN 2525-3409 | DOI: http://dx.doi.org/10.33448/rsd-v10i6.15874

A indústria corporal através dos meios de comunicação encarrega-se de criar desejos e reforçar imagens, padronizando corpos. Corpos que se veem fora de medidas, sentem-se cobrados e insatisfeitos. O reforço dado pela mídia em mostrar corpos atraentes, faz com que uma parte de nossa sociedade se lance na busca de uma aparência física idealizada. Podemos associar hoje, o corpo à ideia de consumo. Em muitos momentos este corpo é objeto de valorização exagerada dando oportunidade de crescimento no "mercado do músculo" e ao consumo de bens e serviços destinado à "manutenção deste corpo". E segundo Fernandes (2003, p.13): "O corpo está em alta! Alta cotação, alta produção, alto investimento... Alta frustração".

Pesquisas recentes têm revelado prevalências elevadas de insatisfação com a imagem corporal em adolescentes do sexo feminino, sendo relatados valores de 67,6\% (Graup et al., 2008) e 85\% (Corseuil et al., 2009). Esse quadro tem sido identificado como o principal precursor de transtornos alimentares (TA), como a anorexia e a bulimia nervosa (Erickson et al., 2007). O número de casos desses transtornos na adolescência tem aumentado nos últimos anos, ocorrendo em idades cada vez mais precoces, tornando-se um sério problema de saúde pública.

De acordo com Bittar (2020) Transtornos Alimentares (TA) são doenças psiquiátricas caracterizadas por alterações graves no comportamento alimentar, sendo indispensável e de suma importância à abordagem da terapia nutricional. O trabalho do nutricionista na área de TA exige habilidades não inerentes à sua formação, como conhecimentos de psicologia, psiquiatria e das técnicas de terapia cognitivo-comportamental. Objetivos reais de peso, com incorporação de exercícios físicos regulares, padrão alimentar adequado e mudanças comportamentais para manter o peso corporal adequado e estável são metas do tratamento nutricional dos TA. A anamnese alimentar e a avaliação antropométrica são utilizadas como guias do processo de diagnóstico e do plano de tratamento para TA.

A bulimia nervosa é o transtorno alimentar mais frequente na população e é caracterizada por episódios do comer compulsivo seguido de métodos compensatórios e inadequados para evitar o ganho de peso. É o quadro mais prevalente dentro dos Transtornos Alimentares, sendo uma doença quase que exclusiva em jovens do sexo feminino. Seus principais sintomas são: vômito autoinduzido, edemas, períodos de restrição alimentar e compulsão alimentar. O vômito autoinduzido é muito comum, sendo encontrado em até $95 \%$ dos pacientes.

Na literatura, bulimia, como sintoma, é conhecida há séculos, mas o reconhecimento da mesma como síndrome é de origem recente, aparecendo por volta de 1940 (Casper, 1983). A etiologia da bulimia nervosa não está totalmente esclarecida, mas, de acordo com alguns autores, acredita-se que a baixa autoestima, a depressão, a pressão social para ser magro e a insatisfação com a imagem corporal podem ser fatores que, em associação, contribuem para a ocorrência da bulimia nervosa (Affenito \& Kerstetter, 1999; Thompson \& Chad, 2000; Gucciardi et al., 2004). Este transtorno alimentar pode ser subdividido em bulimia nervosa purgativa, onde o indivíduo faz uso de laxantes e diuréticos e pratica autoindução de vômitos; e em bulimia nervosa não purgativa, onde o indivíduo faz uso de outros métodos compensadores inadequados, tais como a prática de exercícios excessivos e o jejum. O episódio bulímico refere-se á ingestão de uma quantidade excessiva de alimentos, que não visa apenas a saciar uma fome exagerada, mas atende a uma série de estados emocionais ou situações estressantes. Esses episódios vêm sendo referidos como episódios de compulsão alimentar, tratando-se de ingestão alimentar excessiva e descontrolada, geralmente secreta e rápida. Nesses episódios os pacientes só param a ingestão por mal-estar físico, interrupção externa (presença de outra pessoa) ou por esgotamento dos alimentos.

Como principais efeitos dessas práticas, observam-se a perda de fluidos e eletrólitos durante a purgação (desidratação), desequilíbrio acidobásico, desequilíbrio eletrolítico, arritmias cardíacas, diminuição na concentração de potássio sérico (principal cátion responsável pela contração muscular), distúrbios gastrintestinais, aumento da glândula parótida, erosão e perda de esmalte dentário (Beatty \& Finn, 1995; Villardi et al., 2000). Diferentemente do anoréxico, cuja magreza extrema põe em evidência a existência de um problema, o bulímico possui peso normal ou leve sobrepeso; por isso, a doença pode manter-se oculta durante anos (Morandé \& Carreira, 1988; Yeager et al., 2000). 
Em atletas, embora a prevalência de anorexia nervosa e bulimia nervosa ainda não sejam suficientemente conhecidas, sobretudo no Brasil, pesquisas realizadas demonstraram frequência aumentada (de 15 a $62 \%$ ), sobretudo em certas modalidades desportivas (Ruud, 1996). A estreita relação entre imagem corporal e desempenho físico faz com que as atletas sejam um grupo particularmente vulnerável à instalação desses transtornos, tendo em vista a ênfase dada ao controle de peso (Oliveira et al., 2003). Estudos recentes citam a influência exercida pelos treinadores, patrocinadores e familiares, por meio de seus comentários relativos ao peso e à forma das atletas, como um poderoso elemento de instalação de comportamentos alimentares anormais. Sabe-se, ainda, que a adoção de dietas restritivas em idade precoce, sobretudo, se essa prática se dá sem a supervisão de um profissional, pode desencadear TCA (transtornos de comportamento alimentar), causando danos importantes à saúde e, por conseguinte, ao desempenho atlético (Mclean et al., 2001; COBB et al., 2003).

Esportes que preconizam o baixo peso corporal e supervalorizam a estética, utilizando-a como critério para a obtenção de bons resultados em competições - como ocorre, por exemplo, na ginástica artística, natação sincronizada, corrida e no balé -, têm sido indicados, por pesquisas realizadas nessa área, como os de maior incidência de TCA e de comportamentos considerados precursores de TCA (Lopiano \& Zotos, 1992; Rodin \& Larson, 1992; Sundgot-Borgen, 1994).

A ginástica olímpica é um esporte caracterizado pela leveza dos movimentos, pela arte do equilíbrio, flexibilidade e pelo domínio do corpo. Devido a isso se preconiza que ginastas sejam mais leves e mais magras do que outras meninas da mesma idade. Em se tratando de corridas de longa distância, atletas que apresentam um menor peso corporal e diminuída percentagem de gordura corpórea estariam em vantagem em relação às outras, pois neste esporte a atleta tem que conduzir seu corpo a uma longa distância em velocidade máxima (Bale, 1994).

West (1998), analisando 42 atletas universitárias de ginástica, verificou que 62\% estavam usando pelo menos um tipo de prática alimentar inadequada. A prática de restrição alimentar, que já não é recomendada a jovens não atletas, é extremamente prejudicial para atletas, pois pode levar a um déficit calórico e ao desequilíbrio eletrolítico, que têm como consequência a desnutrição e o comprometimento do desempenho físico. A dieta hipocalórica está também associada à baix a do pulso e da frequência do hormônio luteinizante e do tiidotironina (Loucks et al., 1998). Esses hormônios atuam no controle do ciclo menstrual e, quando alterados, promovem irregularidades, como a amenorreia secundária e a oligomenorréia, sintomas típicos de TCA. A inter-relação e, em muitos casos, a coexistência de TCA, amenorréia e osteoporose caracterizam a tríade da mulher atleta (Safran et al., 2002). Segundo a literatura, a prevalência da tríade da mulher atleta varia de 10 a $66 \%$ entre atletas de alto nível, sobretudo, nas modalidades em que a baixa massa corporal é preconizada (corredoras, ginastas, bailarinas) (Safran et al., 2002; Yeager et al., 1993).

O desenvolvimento de padrões alimentares anormais pode levar à disfunção menstrual e, consequentemente, à osteoporose, sendo, portanto, necessário o controle dos sintomas dessas alterações. Cada componente dessa tríade pode interferir no desempenho físico, e, ainda, causar morbidade ou mesmo mortalidade.

A busca dos adolescentes por um corpo perfeito está gerando excessos e preocupando profissionais da área de saúde e do desporto, mas por outro lado relatos vêm sendo apresentados em que profissionais de Educação Física não estão estabelecendo limites a seus alunos, nem mesmo distinguindo uma prática saudável de um exercício obsessivo.

Os impactos positivos da prática regular de atividade física para a saúde são evidenciados na literatura por meio de estudos epidemiológicos (Matsudo, 2001). Atividade física é qualquer movimento corporal produzido pelos músculos esqueléticos que resulta em um gasto energético maior do que os níveis de repouso. No entanto, indivíduos com transtornos alimentares frequentemente não se beneficiam, na medida em que podem utilizá-los como estratégia para perder peso de forma inadequada e por vezes de forma compulsiva. Sugere-se que aproximadamente 55\% dos pacientes com bulimia nervosa não purgativa (BN) praticam exercício físico de forma compulsiva em algum momento de sua história clínica (peñas-lledó et al., 2002; DAVIS, C., 1997). 
Além dos clássicos distúrbios, uma condição prevalente entre atletas é a "Anorexia Atlética". Os critérios para seu diagnóstico incluem: perda de peso, atraso na puberdade, disfunção menstrual, queixas gastrintestinais, ausência de doença ou desordem afetiva que pudesse explicar a redução de peso, falsa imagem corporal, excessivo medo de ganhar peso, restrição alimentar, vômitos autoinduzidos, uso de laxantes, diuréticos e exercícios físicos compulsivos (Sundgot-Borgen, 1994).

A maioria das atletas que querem perder peso não apresentam conhecimentos suficientes sobre nutrição e métodos apropriados de controle ponderal e não seguem uma orientação ou supervisão, adquirindo informações de outras atletas, treinadores ou revistas não especializadas. Loosli et al. (1986) realizaram um estudo com 97 ginastas adolescentes competitivas, onde classificaram como inadequados os conhecimentos nutricionais das ginastas, observando a importância de programas de educação nutricional para o grupo estudado. As informações foram obtidas através de um questionário que envolveu questões sobre composição dos alimentos e como tais alimentos se relacionavam com o desempenho atlético.

Ribeiro (1995) realizou um estudo com 54 ginastas adolescentes, pertencentes a clubes e academias da cidade do Rio de Janeiro e São Paulo com o objetivo de avaliar o consumo alimentar de atletas de ginástica olímpica. Os métodos de avaliação dietética utilizados foram: recordatório de 24 horas e o registro alimentar de 3 dias. Os resultados demonstraram que o consumo energético das atletas cariocas e paulistas, de 11 a 14 anos, estava abaixo das recomendações.

Estudos mostram que atletas, especialmente as de resistência, tendem a desenvolver uma condição conhecida como "anemia esportiva" ou "pseudoanemia", que pode ocorrer no início de um programa de treinamento ou durante um treinamento prolongado. Reduções transitórias na concentração de hemoglobina, na contagem de hemácias e hematócrito, que caracterizam esta condição, podem ser devidas à expansão do volume plasmático como uma adaptação fisiológica aos exercícios extenuantes (Nachtigall et al., 1996). Tal aumento no volume plasmático pode ser benéfico devido ao decréscimo na resistência ao fluxo sanguíneo, melhora na sudorese e um maior volume de ejeção (Haymes, 1996). Entretanto, pesquisas com atletas de resistência, do sexo feminino, revelam que estas não apresentam apenas uma redução na concentração de hemoglobina relacionada a uma hemodiluição, mas também um estado de deficiência de ferro com ou sem anemia. Atletas femininas que consomem dietas com baixa biodisponibilidade de ferro, como por exemplo, dietas vegetarianas, podem se encontrar em risco de uma deficiência devido à baixa ingestão de ferro heme (Nuviala \& Lapieza, 1997). A perda de ferro através da transpiração excessiva também pode estar relacionada ao balanço negativo deste mineral. Atletas que treinam exaustivamente podem eliminar 1 a 2 litros de suor por hora, perdendo também uma quantidade substancial de ferro (Telford et al.,1993). Contudo, parte do ferro perdido no suor pode ser proveniente da descamação cutânea (Haymes, 1996).

Atletas femininas que praticam exercícios físicos intensos e apresentam alterações no comportamento alimentar, comumente apresentam irregularidades menstruais caracterizadas por oligomenorreia e amenorreia. O equilíbrio endócrino que regula as funções reprodutoras femininas pode ser alterado por fatores genéticos, psicológicos e físicos. O treinamento físico intenso pode estar associado à presença de irregularidades menstruais. Atletas femininas que desempenham exercícios físicos intensos são as que mais apresentam tais irregularidades. Muitos fatores encontram-se associados à amenorreia atlética. Os frequentemente citados são: distúrbios no comportamento alimentar, restrição dietética, intensidade dos treinamentos, estresse psicológico e fisiológico, treinamento físico antes da menarca, retardo do início da função menstrual, baixo peso corporal e baixo percentual de gordura corpórea (Bale, 1994; Ruud \& Grandjean, 1996).

Osteoporose é uma condição clínica definida como um decréscimo na massa óssea (matriz osteóide e componente microcristalino inorgânico) (Putukian, 1994). Associa-se a redução na densidade minerálica óssea principalmente da coluna vertebral e do fêmur proximal e a maior incidência de injúrias musculoesqueléticas (Putukian, 1994; Ruud \& Grandjean, 1996).

Acredita-se que a amenorreia é o principal fator de risco para a redução da massa óssea (Wilmore, 1991). São achados frequentes em atletas amenorreicas: incapacidade de alcançar o pico de massa óssea, perda prematura de massa óssea e osteoporose. 
Em pacientes com bulimia nervosa a terapia nutricional tem como objetivo a normalização do padrão alimentar por meio da redução e/ou cessação do ciclo de restrição/compulsão alimentar/métodos compensatórios. Estes pacientes possuem dietas caracterizadas em ciclos de restrição/ compulsão/ purgação, tornando o comportamento alimentar caótico (Ada, 2001). É importante esclarecer para o paciente a conduta nutricional que será adotada em relação às mudanças no comportamento, padrão alimentar, adequação do peso, uso do diário alimentar (método no qual o paciente registra toda a sua ingestão alimentar efetuada, assim como seus sentimentos no momento da alimentação.) e estabelecimento de um plano alimentar regular. Também deve ser discutido com o paciente qual é o seu peso ideal, com base na sua compleição física e história de peso. O paciente deve compreender que o peso ideal é determinado em função da sua saúde, e não de padrões de beleza ou de exigências pessoais desmedidas (Latterza et al., 2004). A intervenção nutricional deverá ser gradativa e por etapas, de acordo com as prioridades detectadas por meio de diário alimentar. Introduzem-se, portanto, tarefas objetivas para a mudança do comportamento alimentar e a cessação dos métodos compensatórios, como estabelecer horários para as refeições, não omitir refeições, mastigar bem os alimentos, visualizar as porções servidas das refeições e dos lanches que serão consumidos, evitar os "beliscos" e não comer na frente da televisão.

\section{Considerações Finais}

A presença de anorexia ou bulimia nervosa entre as atletas representa uma preocupação com o peso corporal associada à leve distorção da imagem corporal e à prática de dietas restritivas. Essas práticas são denominadas síndromes parciais do TCA e, quando associadas a exercícios físicos sistemáticos e extenuantes, são fatores de risco, cuja detecção precoce pode impedir a instalação futura dos quadros graves de TCA.

Como as atletas e seus treinadores não apresentam conhecimentos adequados sobre nutrição, geralmente as atletas comprometem a própria saúde esforçando-se para alcançarem ou manterem uma meta inadequada de peso corpóreo, ou seja, um percentual de gordura corporal tão baixo quanto possível.

Devido a isso, torna-se necessário uma educação nutricional direcionada às atletas, seus treinadores e à equipe de apoio para que os mesmos se conscientizem dos efeitos adversos dos métodos extremos de controle de peso sobre a saúde destas próprias atletas.

Porém faz-se necessário pesquisas futuras referentes ao tema do presente artigo assim como maiores investigações sobre o impacto, a longo prazo, da bulimia em adolescentes que praticam qualquer tipo de atividade física.

\section{Referências}

Ada - American Dietetic Association. (2001) - Position of the American Dietetic Association: nutrition intervention in the treatment of anorexia nervosa, bulimia nervosa and eating disorders not otherwise specified (EDNOS). J Am Diet Assoc 101: 810- 9.

Affenito, S.G.; Kerstetter, J. (1999) Position of the American Dietetic Association and Dietitians of Canada: women's health and nutrition. J. Am. Diet. Assoc., Chicago, v. 99 , n. 6, p. 738-751.

Assunção, S.S.M.; Cordás, T.A.; Araújo, L.A.S.B.;(2002) Atividade física e transtornos alimentares. Revista de Psiquiatria Clínica, v. 29, p. 4-13

Bale, P. (1994) Body composition and menstrual irregularities of female athletes. Are they precursors of anorexia? Sports Medicine, Auckland, v.17, n.6, p.347352.

Barreto, A.L.H.; FIGUEIRÓ, A.S.; SOARES, R.M. Padrão alimentar dos transtornos alimentares. In: nunes, m.a.; Appolinario, j.c.; Galvão, a.l.; Coutinho, W. Transtornos alimentares e obesidade. $2^{\mathrm{a}}$ ed. São Paulo: Artmed, 2006, p. 117-124.

Baudrillard, J. (1991) The Transfer of Bad Essay on External Phenomena. Barcelona: Anagrama

Beatty, D. \& Finn, S.C. (1995) Position of the American Dietetic Association and the Canadian Dietetic Association: women's health and nutrition. J Am Diet Assoc 95:362-6.

Bittar, C., \& Soares, A. (2020). Mídia e comportamento alimentar na adolescência. Cadernos Brasileiros de Terapia Ocupacional, $28(1), 291-308$. 
Research, Society and Development, v. 10, n. 6, e29110615874, 2021

(CC BY 4.0) | ISSN 2525-3409 | DOI: http://dx.doi.org/10.33448/rsd-v10i6.15874

Carvalho, R.S.; Amaral, A.C.S.; Ferreira, M.E.C.; (2009) Transtornos alimentares e imagem corporal na adolescência: uma análise da produção científica em psicologia. Psicologia: Teoria e Prática, v. 11, p. 200-223.

Castilla, D.; Bastin, C. (1998) La Boulimie: mieux se connaître pour en guérir. Paris: Éd. Robert Laffont ; .

Castro, J.M.; Goldstein, S.J. (1995) Eating attitudes and behaviors of pre- and pos pubertal females: clues to the etiology of eating disorders. Physiol Behav $58(1): 15-23$.

Claudino, A. de M.; BorgeS, M.B.F. (2002) Critérios diagnósticos para os transtornos alimentares: conceitos em evolução. Revista Brasileira de Psiquiatria, São Paulo, v. 24, p. 7-12. Suplemento III.

Cobb, K.L.; Bachrach, L.K.; Greendale, G.; Marcus, R.; Neer, R.M.; Nieves, J. (2003) Disordered eating, menstrual irregularity, and bone mineral density in female runners. Med Sci Sports Exerc 35:711-9.

Constantini, N.W. (1994) Clinical consequences of athletic amenorrhea. Sports Medicine, Auckland, v.17, n.4, p. 213-223, 1994.

Cordás, T.A.; Salzano, F.T.; Rrios, S.R. (2004) Os transtornos alimentares e a evolução no diagnóstico e no tratamento. In: PHILIPPI, S. T.; ALVARENGA, M. Transtornos alimentares: uma visão nutricional. São Paulo: Manole. p. 39-62.

De Jesus, VM, Ferreira, JMA e da Silva Lima, W. (2018). A auto percepção estética na sociedade moderna. Anais da Jornada de Educação Física do Estado de Goiás (ISSN 2675-2050), 1 (1), 135-139.

Dos Santos, CR (2019). Imagem corporal e distúrbios alimentares: análise das opiniões de alunos do ensino médio. Revista Multidisciplinar da Saúde, 1 (1), 28 42 .

Dunker, K.L.L; Fernandes, C.P.B.; Filho, D.C. (2009) Influência do nível socioeconômico sobre comportamentos de risco para transtornos alimentares em adolescentes. J Bras Psiquiatr, v. 58, p. 156-161.

Estrela, C. (2018). Metodologia Científica: Ciência, Ensino, Pesquisa. Editora Artes Médicas.

Fernandes, M. H. (2003). Corpo. Clínica Psicanalítica. São Paulo: Casa do Psicólogo.

Figueiredo, R. (2019). A obsessão pelo corpo e o desenvolvimento de transtornos alimentares em jovens adolescentes. Pretextos-Revista da Graduação em Psicologia da PUC Minas, 4(7), 250-268.

Galvão, a.1.; Claudino, A. de M.; Borges, M.B.F. (2006) Aspectos e evolução do diagnóstico. In: Nunes, M.A.; Appolinario, J.C.; Galvão, A.L.; Coutinho, W. Transtornos alimentares e obesidade. $2^{\mathrm{a}}$ ed. São Paulo: Artmed, p. 31-50.

Graup, S.; Ppereira, E.F.; Lopes, A.S.; Araújo, V.C.; LegnanI, R.F.S; Borgatto, A.F. (2008) Associação entre a percepção da imagem corporal e indicadores antropométricos de escolares. Revista Brasileira de Educação Física e Esporte, São Paulo, v. 22, p. 129-38.

Haymes, E.M. (1996) Minerais de traço e exercícios. In: Wolinsky, I., Hickson JR, J.F. Nutrição no exercício e no esporte. 2.ed. São Paulo : Roca, p.242-257, .

Kaneshima, A.M. de S.; França, A.A.; Kneube, D. de P.F.; Kaneshima, E.N. (2008) Identificação de distúrbios da imagem corporal e comportamentos favoráveis ao desenvolvimento da bulimia nervosa em adolescentes de uma Escola Pública do Ensino Médio de Maringá, Estado do Paraná. Acta Sci. Health Sci, Maringá, v. 30, n. 2 , p. $167-173$.

Koche, J. C. (2011). Fundamentos de metodologia científica. Petrópolis: Vozes. Disponível em: http://www.brunovivas.com/wpcontent/uploads/sites/10/2018/07/K\%C3\%B6che-Jos\%C3\%A9-Carlos0D0AFundamentos-de-metodologia-cient\%C3\%ADfica-_teoriada0D0Aci\%C3\%AAncia-e-inicia\%C3\%A7\%C3\%A3o-\%C3\%A0-

pesquisa.pdfhttps://repositorio.ufsm.br/bitstream/handle/1/15824/Lic_Computacao_Metodologia-Pesquisa-Cientifica.pdf?sequence=1

Lopiano, D.A.; Zotos C.(1992) Modern athletics, the pressure to perform. In: Brownell KD, Rodin J, Wilmore JH, editors. Eating, body weight and performance in athletes: disorders of modern society. Philadelphia: Lea and Febiger, 275-92.

Loosli, A.R., Benson, J., Gillien, D.M. \& Bourdet, K. (1986) Nutrition habits and knowledge in competitive adolescent female gymnasts. The Physician and Sportsmedicine, v.14, n.8, p.118-130.

Loucks, A.B.; Mortola, J.F.; Girton, L.; Yen, S.S.C. (1998) Alterations in the hypothalamic-pituitary-ovarian and hypothalamic-pituitary-adrenal axes in athletic women. J Clin Endocrinol Metab 68:402-11

Ludke, M. \& Andre, M. E . D. A. (2013). Pesquisas em educação: uma abordagem qualitativa. São Paulo: E.P.U.

Maldonado, G. de R. (2006) A educação física e o adolescente: a imagem corporal e a estética da transformação na mídia impressa. Revista Mackenzie de Educação Física e Esporte, v. 5, p. 59-76.

Matsudo, S. M. M. (2001) Envelhecimento \& atividade física. Londrina: Midiograf. 195p.

Mclean, J.A.; Barr, S.I.; Prior, J.Y.C. (2003) Dietary restraint, exercise, and bone mineral density in young women: are they related? Med Sci Sports Exerc; 33:1292-6. 
Moehlecke, M., Blume, CA, Cureau, FV, Kieling, C., \& Schaan, BD (2020). Auto-imagem corporal, insatisfação com o peso corporal e estado nutricional de adolescentes brasileiros: um estudo nacional. Jornal de Pediatria, 96 (1), 76-83.

Morandé, G \& Carreira, M. (1988) Anorexias nerviosas y bulimias. Una epidemia actual en adolescentes? Algunos aspectos en discusión. Revista de Psiquiatría de Chile; V:19-28

Murari, K. S., \& Dorneles, P. P. (2018). Uma revisão acerca do padrão de autoimagem em adolescentes. Revista Perspectiva: Ciência e Saúde, 3(1), 155-168.

Nachtigalll, P., Nielsen, P., Fisher, R., Engelhardt, R., Gabbe, E.E. (1996) Iron deficiency in distance runners: a reinvestigation using Fe-labelling and noninvasive liver iron quantification. International Journal of Sports Medicine, Stuttgart, v.17, n.7, p.473-479.

Nunes, M. A.; Bagatini, L. F.; Abuchaim AL, A. L.; Kunz, A.; Ramos, D.; Silva, J. A.; Somenzi, L.; Pinheiro, A. (1994) Distúrbios da conduta alimentar: considerações sobre o Teste de Atitudes Alimentares (EAT). Revista ABPAPAL, São Paulo, v. 1, p. 7-10.

Nuviala, R.J., Lapieza, M.G. (1997) Disparity between diet and serum ferritin in elite sportswomen. Nutrition Research, v.17, n.3, p.451-461.

Oliveira, F.P.; Bosi, M.L.M.; Vigário, P.S.; Vieira, R.S. (2003) Comportamento alimentar e imagem corporal em atletas. Rev Bras Med Esporte, 9(6): 348-356.

Pereira A. S. et al. (2018). Metodologia da pesquisa científica. [free e-book]. Santa Maria/RS. Ed. UAB/NTE/UFSM.

Putukian, M. (1994) The female triad: eating disorders, amenorrhea and osteoporosis. Medical Clinics of North America, Philadelphia, v.78, n.2, p.345-356.

Ribeiro, B.G. (1995) Avaliação nutricional de ginastas competitivas de ginástica olímpica. Rio de Janeiro, 1995. 110p. Dissertação (Mestrado em Nutrição Humana) - Instituto de Nutrição, Universidade Federal do Rio de Janeiro.

Rodin, J. \& Jarson, L. (1992) Social factors and the ideal body weight and the ideal body shape. In: Brownell KD, Rodin J, Wilmore JH, editors. Eating, body weight and performance in athletes: disorders of modern society. Philadelphia: Lea and Febiger; 146-58;

Russo, R. (2005) Imagem corporal: construção através da cultura do belo. Movimento \& Percepção, SP, v.5, n.6

Ruud, J.S. (1996) Nutrition and the female athlete. New York, NY: CRC Press.

Ruud, J.S., Grandjean, A.C. (1996) Preocupações Nutricionais das Atletas. In: Wolinsky, I., Hickson JR, J.F. Nutrição no exercício e no esporte. 2.ed. São Paulo.:.Roca, p.379-398.

Safran, M.R.; Mckeag, D.B.; Camp, S.P.V. (2002) Manual de medicina esportiva. São Paulo, SP: Manole.

Scherer, F.C.; Martins, C.R.; Pelegrini, A.; Matheus, S.C.; Petroski, E.L. (2010) Imagem corporal em adolescentes: associação com a maturação sexual e sintomas de transtornos alimentares J Bras Psiquiatr, v. 59, p. 198-202.

Schmitz, R.J.; Alves, A.M.P. (2014) Bulimia na adolescência: a visão de profissionais da saúde. Revista UEPG Ciências Biológicas e da Saúde, Ponta Grossa, v. 15 , n. 1, p. 19-31.

Silva, I. S. D. S., Pontes, E. D. S.,Silva, M. C. C., \& Silva, E. C. A. (2018). Adolescente e Imagem Corporal: uma Revisão. International Journal of Nutrology, $11(1)$.

Sundgot-borgen, J. (1994) Risk and trigger factors for the development of eating disorders in female elite athletes. Med Sci Sports Exerc 26: 414-9.

TavareS, M.C.C. (2003). Imagem Corporal: Conceito e Desenvolvimento. São Paulo: Manole.

Teixeira, P.C.; Costa, R.F.; Matsudo, S.M.M.; Cordás, (2009) T.A. A prática de exercícios físicos em pacientes com transtornos alimentares. Revista de Psiquiatria Clínica, São Paulo, v. 36 n. 4.

Telford, R.D., Cunningham, R.B., Deakin, V., Kerr, D.A. (2000) Iron status and diet in athletes. Medicine and Science in Sports and Exercise, Baltimore, v.25, n.7, p.796-800, 1993. THOMPSON \& CHAD.

Vale AMO. (2002) Prevalência de comportamentos alimentares anormais e práticas inadequadas de controle de peso entre estudantes secundaristas da rede pública e privadas de Fortaleza (dissertação de mestrado) Ceará: Faculdade de Medicina da Universidade Federal do Ceará.

Villardi, T.C.C.; Ribeiro, B.G.; Soares, E.A. (2000) Distúrbios nutricionais em atletas femininas e suas inter-relações. (2000) Revista de Nutrição 1:61-9.

West, R.V. (1998) The female athlete. The triad of disordered eating, amenorrhea and osteoporosis. Sports Med 26:63-71.

Wilmore, J.H. (1991) Eating and weight disorders in the female athlete. International Journal of Sport Nutrition, Champaign, v.1, n.2, p.104-117.

Yeager, K.K.; Agostini, R.; Nattiv, A.; Drinkwater, B. (1993) The female athlete triad - disordered eating, amenorrhea and osteoporosis. Med Sci Sports Exerc 25:775-7.

Yeager, J.; Andersen, A.; Devlin, M.; Egger, H.; Herzog, D.; Mitchell, J. (2000) Practice guideline for the treatment of patients with eating disorders. $2^{\text {nd }}$ ed. In: American Psychiatric Association. Practice guidelines for treatment of psychiatric disorders: compendium 2000. 1st ed. Washington, DC: American Psychiatric Press, 121-6.

Yin, R.K. (2015). O estudo de caso. Porto Alegre: Bookman 